\title{
Adverse Renal Effects of Immune Checkpoint Inhibitors: A Narrative Review
}

\author{
Rimda Wanchoo ${ }^{a}$ Sabine Karam ${ }^{c}$ Nupur N. Uppal ${ }^{a}$ Valerie S. Barta ${ }^{a}$ \\ Gilbert Deray $^{d}$ Craig Devoe $^{b}$ Vincent Launay-Vacherd, e Kenar D. Jhaveri ${ }^{a}$ \\ on behalf of Cancer and Kidney International Network Workgroup on Immune \\ Checkpoint Inhibitors

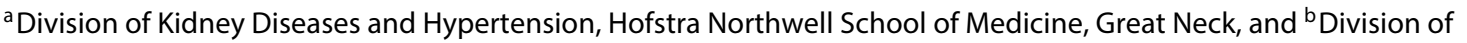 \\ Hematology and Oncology, Hofstra Northwell School of Medicine and Northwell Cancer Institute, New Hyde Park, USA; \\ 'Department of Medicine, University of Balamand, Faculty of Medicine, Beirut, Lebanon; d Nephrology Department, \\ Pitié-Salpêtrière University Hospital, and ' Service ICAR, Pitié-Salpêtrière University Hospital, Paris, France
}

\section{Key Words}

Renal failure - Acute interstitial nephritis · Ipilimumab · Nivolumab · Onconephrology · Pembrolizumab · Targeted therapies

\begin{abstract}
Background: Cancer immunotherapy, such as anti-cytotoxic T-lymphocyte-associated protein 4 (CTLA-4) and anti-programmed death 1 (PD-1), has revolutionized the treatment of malignancies by engaging the patient's own immune system against the tumor rather than targeting the cancer directly. These therapies have demonstrated a significant benefit in the treatment of melanomas and other cancers. Summary: In order to provide an extensive overview of the renal toxicities induced by these agents, a Medline search was conducted of published literature related to ipilimumab-, pembrolizumab-, and nivolumab-induced kidney toxicity. In addition, primary data from the initial clinical trials of these agents and the FDA adverse reporting system database were also reviewed to determine renal adverse events. Acute interstitial nephritis (AIN), podocytopathy, and hyponatremia were toxicities caused by ipilimumab. The main ad-
\end{abstract}

verse effect associated with both the PD-1 inhibitors was AIN. The onset of kidney injury seen with PD-1 inhibitors is usually late (3-10 months) compared to CTLA-4 antagonists related renal injury, which happens earlier (2-3 months). PD-1 as opposed to CTLA-4 inhibitors has been associated with kidney rejection in transplantation. Steroids appear to be effective in treating the immune-related adverse effects noted with these agents. Key Message: Although initially thought to be rare, the incidence rates of renal toxicities might be higher (9.9-29\%) as identified by recent studies. As a result, obtaining knowledge about renal toxicities of immune checkpoint inhibitors is extremely important.

(c) 2017 S. Karger AG, Basel

\section{Introduction}

Immune checkpoint inhibitors (ICI) are increasingly being used in the treatment of several malignancies. Immune surveillance of cancer occurs through the activity of both the innate and adaptive immune systems, whereby malignant cells are detected and eliminated in their earliest stages [1]. However, tumors escape this process

\section{KARGER}

(c) 2017 S. Karger AG, Basel

E-Mail karger@karger.com

www.karger.com/ajn
Kenar D. Jhaveri, MD

Professor of Medicine, Nephrology, Northwell Health

Hofstra Northwell School of Medicine

Great Neck, NY 11021 (USA)

E-Mail kjhaveri@northwell.edu 


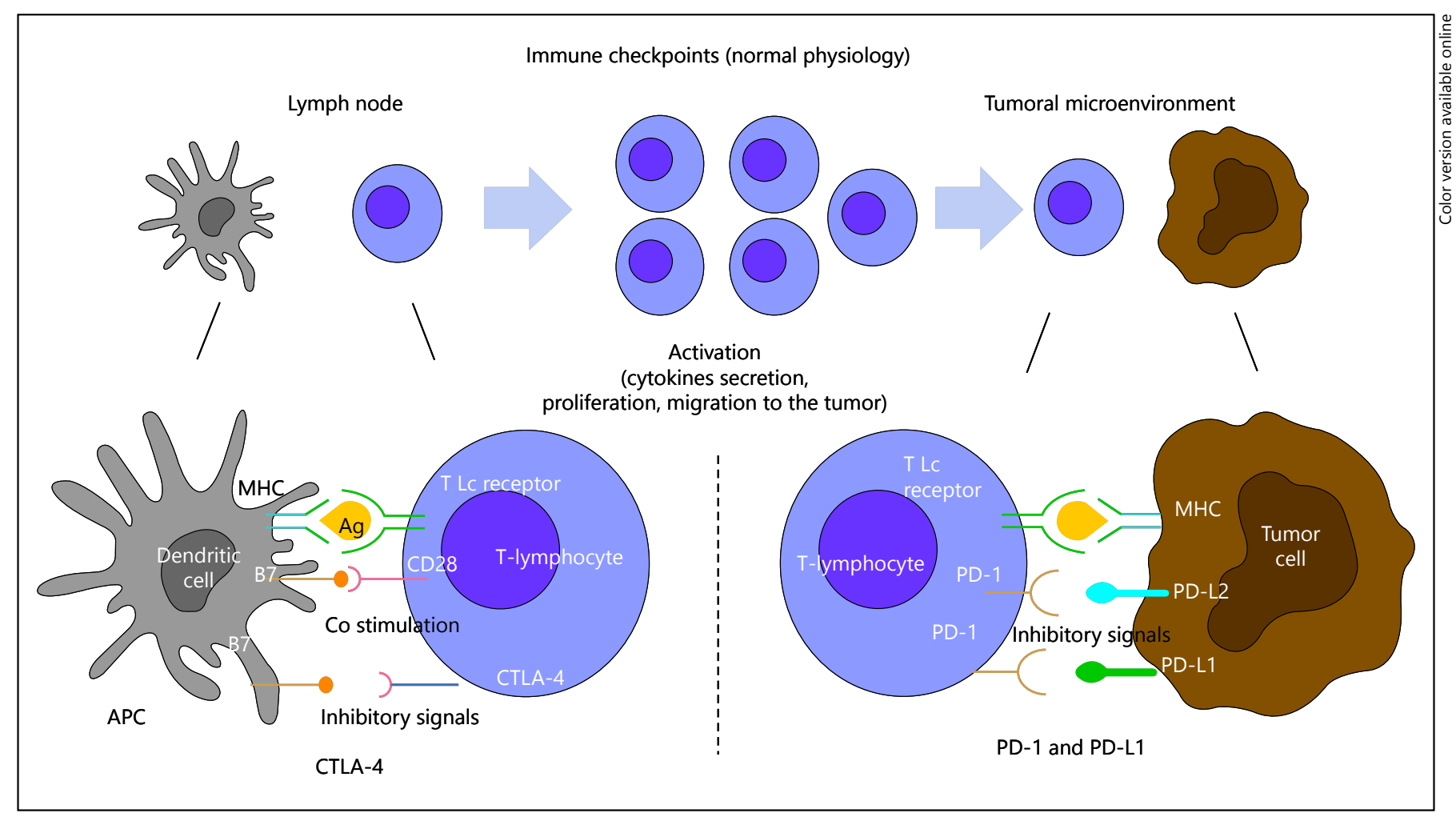

Fig. 1. CTLA-4 and PD-1 signaling networks at homeostasis. Integration of both positive and negative costimulatory signals during and after the initial T-cell activation will determine the fate and intensity of the alloimmune response. The first step in antigen (Ag) recognition is the binding of the antigen to major histocompatibility complex (MHC) molecules on the antigen presenting cell (APC) and creating a complex with the T cell receptor (TCR) located on the $\mathrm{T}$ cell. This is followed by the interaction of the CD28 molecule with B7 (CD 80/86) initiating a co-stimulatory signal leading to further $\mathrm{T}$-cell stimulation (this is in addition to other co-stimulatory molecules not depicted here). As a negative

by employing several mechanisms to avoid or actively suppress anticancer immune responses $[2,3]$. Enhancing anti-tumor T-cell immunity with checkpoint inhibitor antibodies such as anti-cytotoxic T-lymphocyte-associated protein 4 (CTLA-4) and anti-programmed death 1 (PD-1) has shown significant clinical benefits in tumor regression and prolonged stabilization of many solid tumors and is now FDA approved for use in non-small cell lung cancer, melanoma, and renal cell cancer. Figure 1 describes CTLA-4 and PD-1 signaling networks in homeostasis and Figure 2 summarizes the mechanism of action of CTLA-4 and PD-1 antagonists in malignancy. The T-cell receptor (TCR) interacts with the major histocompatibility complex in association with antigen on the antigen presenting cell (APC). The cell surface mol- feedback process to prevent overstimulation, T-cell activation leads to the upregulation of the CTLA-4 molecule, which competes with the B7-CD28 ligand and in turn leads to T-cell arrest, thus providing brakes to the immune system. Similarly, binding of the PD-1 molecule with PD-L1 and PD-L2 leads to an inhibitory signal with decreased effector T-cell function, suppressing immune surveillance and permitting neoplastic growth. It has to be noted that the majority of data supports the role of increased PD-L1 expression in human tumors and serves as the biomarker to consider PD-1 inhibitors for treatment. The role of PD-L2 in specific tumor immunology in humans is not well defined.

ecule CD28 mediates a positive co-stimulatory signal to the $\mathrm{T}$ cell via interaction with $\mathrm{B} 7$ receptors on the APC. The CTLA- 4 is then upregulated on the T cell, which in turn competes with the B7-CD28 ligand molecule. CTLA-4 upregulation leads to an inhibitory signal and induces T-cell arrest. CTLA-4 activation puts the "brakes" on the immune system [4]. Ipilimumab is a monoclonal antibody that has anti-tumor activity by targeting CTLA4 and activating the immune system. The inhibitory receptor PD-1 is a cell surface molecule with a single immunoglobulin super-family domain. It is expressed on activated $\mathrm{T}$ cells, $\mathrm{B}$ cells, natural killer T cells, monocytes, and dendritic cells [5-7]. PD-1 has 2 ligands: PD-ligand-1 (L1) and ligand-2 (L2). Tumor expression of PD-L1 is thought to be one mechanism of immune 


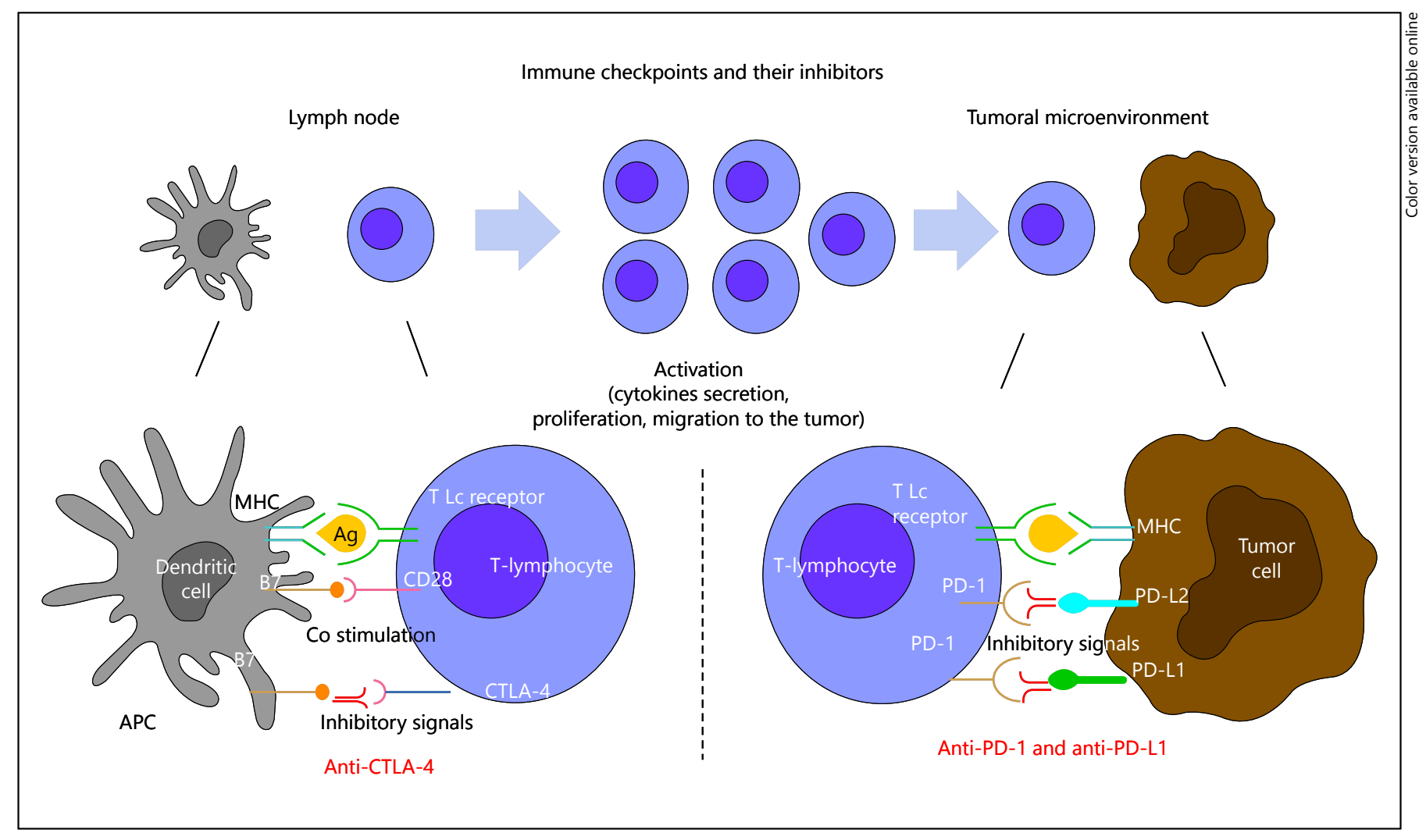

Fig. 2. Ipilimumab or CTLA-4 antagonist binds to the CTLA-4 molecule and prevents it from binding to B7, leading to the sustained activation of the T cell (lifting the foot off the brakes). PD- 1 inhibitors bind to the PD-1 molecule preventing its interaction with $\mathrm{PD}-\mathrm{L} 1 / \mathrm{L} 2$, thus leading to continued T-cell stimulation

evasion by cancer cells. The role of PD-L2 in cancer immunology is far less clear [6, 7]. Monoclonal antibodies directed against PD-1 prevent the engagement of PD-1 with its ligands, leading to $\mathrm{T}$-cell stimulation. They assert anti-neoplastic activity by rescuing the $\mathrm{T}$ cell from apoptotic death, thus allowing it to continue its attack on tumor cells [5]. Nivolumab and pembrolizumab are 2 monoclonal antibody therapies designed to directly block the interaction between PD-1 and its ligands and have been successfully used in treatment of melanoma, lung cancer, renal cancer, and hematological malignancies since 2014 [8-10].

Immune-related toxicities (colitis, dermatitis, pneumonitis, hepatitis, and thyroiditis) are common with ICI $[4,5]$. This narrative review summarizes all published contributions related to ipilimumab-, pembrolizumab-, and nivolumab-induced renal toxicities. In addition, primary data from clinical trials, and FDA adverse reporting system was reviewed [11]. (pressing on the accelerator). It has to be noted that the majority of data supports the role of increased PD-L1 expression in human tumors and serves as the biomarker to consider PD-1 inhibitors for treatment. The role of PD-L2 in specific tumor immunology in humans is not well defined.

\section{Incidence}

To better understand recently published contributions related to ipilimumab-, pembrolizumab-, and nivolumab-induced renal toxicities, a Medline, Embase, and Scopus search of indexed manuscripts was conducted. A total of 17 articles and original investigations that included over 100 patients with renal outcomes secondary to the 3 agents were reviewed. In addition, details of all case reports reviewed can be found in online supplementary Tables 1-5 (for all online suppl. material, see www.karger. com/doi/10.1159/000455014).

Cortazar et al. [12] analyzed data from published phase 2 and 3 clinical trials of patients with adverse renal outcomes and found the overall incidence of acute kidney injury (AKI) to be $2.2 \%$ among a total of 3,695 patients. The incidence of grade III or IV (National Cancer Institute Common Terminology Criteria for adverse events) AKI or need for dialysis was $0.6 \%$ [12]. AKI occurred more frequently in patients who received combi- 
nation therapy with ipilimumab and nivolumab (4.9\%) than in patients who received mono-therapy with ipilimumab $(2.0 \%)$, nivolumab (1.9\%), or pembrolizumab (1.4\%) [12]. A recent abstract reported an incidence of renal events as high as $13.9 \%$ with the use of these agents in routine practice in the United States, being associated with the highest toxicity-induced costs $(\$ 8,854)$ [13]. Our unpublished data [14] analyzed 211 patients who received ipilimumab, nivolumab, or pembrolizumab. Ninety-nine patients had a serum creatinine available for analysis. AKI stage 1 (based on AKI network criteria) developed in $29 \%(11 / 38)$ of patients who were given ipilimumab and in 24.5\% (15/61) of patients who were given PD-1 inhibitors. AKI stage 2 developed in 5\% $(2 / 38)$ of patients who were given ipilimumab and $10 \%$ $(6 / 61)$ of patients who were given PD-1 inhibitors. In summary, while initial studies had quoted a small incidence of AKI with ICI use, recent unpublished emerging data suggest a higher incidence rate of AKI (9.9-29\% range) with ICI.

\section{Ipilimumab}

Ipilimumab is a monoclonal antibody that has antitumor activity by targeting CTLA- 4 and activating the immune system. Cell-mediated immune response leading to inflammatory cell infiltrates with and without granulomas has been reported $[15,16]$. Cases of acute interstitial nephritis (AIN) in the initial trials, arising 2-12 weeks post drug administration have been reported and one of them demonstrated granulomas [12, 17-22]. The largest series of biopsy-proven cases of AKI was reported by Cortazar et al. [12]. Pathology revealed AIN in most cases with varying degrees of foot process effacement. In addition, cases of nephrotic syndrome $[7,19]$ in the form of minimal change disease and membranous nephropathy have been reported. Out of a total of 13 cases that were noted to have renal injury associated with ipilimumab, there was no specific gender predilection noted (online suppl. Table 1). Most of the AKI occurred 6-12 weeks following the start of treatment, with the longest interval being 26 weeks. Eleven out of 13 patients who had AIN or podocytopathy received steroids. Two patients did not receive steroids and had no renal recovery. Of the 11 patients treated, 2 had complete recovery of renal function, 7 patients had partial improvement in renal function, and 2 patients remained dialysis dependent with no improvement. Steroids administered differed in their forms and dosages. Patients who developed AKI earlier in the course responded to steroids in a better way and needed less dialysis.

Adverse Renal Effects of ICIs
Ipilimumab has also been associated with electrolyte disturbances. Two cases of ipilimumab-induced hyponatremia $[23,24]$ due to panhypopituitarism from ipilimumab related hypophysitis have been reported. The incidence of hypophysitis in patients treated with this agent is close to $17 \%$ in clinical trials [23]. Mechanistically, a loss of adrenocorticotrophic hormone-secreting corticotrophs leads to a secondary adrenal insufficiency and loss of regulatory effects of cortisol on arginine vasopressin. This could be the mechanism leading to the hyponatremia. In summary, anti CTLA-4 antagonists may result in AIN, podocytopathy, or hyponatremia related to panhypopituitarism.

\section{Pembrolizumab}

Pembrolizumab [25] is a humanized monoclonal immunoglobulin G4 (IgG4) kappa antibody directed against the PD-1. Initial reports did not mention any renal toxicity [26] associated with pembrolizumab. In recent phase 1 [27] and phase 2 trials [28], AKI in the form of nephritis was reported with an incidence as high as $6.7 \%$ [27]. In the KEYNOTE1 trial, AKI was reported to be present in 2 out of 495 patients and hyperkalemia in 4 patients [10]. A recent study using this agent in lung cancer reported an increase in creatinine in $1.7 \%$ of the patients who received $2 \mathrm{mg} / \mathrm{kg}$ of pembrolizumab and $2 \%$ in patients who received the higher dose, but none of the patients on standard chemotherapy (docetaxel) [29] developed AKI. Online supplementary Table 2 summarizes more recent case reports of renal toxicities caused by this agent $[12,30,31]$. Overall, the 4 cases $[12,31]$ of biopsyproven AIN reported with this agent were in the time frame of 1-12 months. There was no gender preference. Three patients responded to steroids with complete remission and 1 patient required dialysis and had partial remission with steroids. Steroids used were variable from intravenous forms to oral steroids for 1-3 months time frame.

\section{Nivolumab}

In the early phase I trials, nivolumab was thought to be innocuous from a renal standpoint $[9,32,33]$. As reported events accumulated, it is now thought that renal adverse events are frequently associated [34] with nivolumab. In a phase I dose-escalation cohort expansion trial in patients with advanced NSCLC, adverse renal events were reported to be present in 3\% of the cases [35]. Two additional phase 2 trials also reported several renal adverse events $[8,36]$. In another phase 3 study, nivolumab monotherapy was compared with docetaxel monothera- 

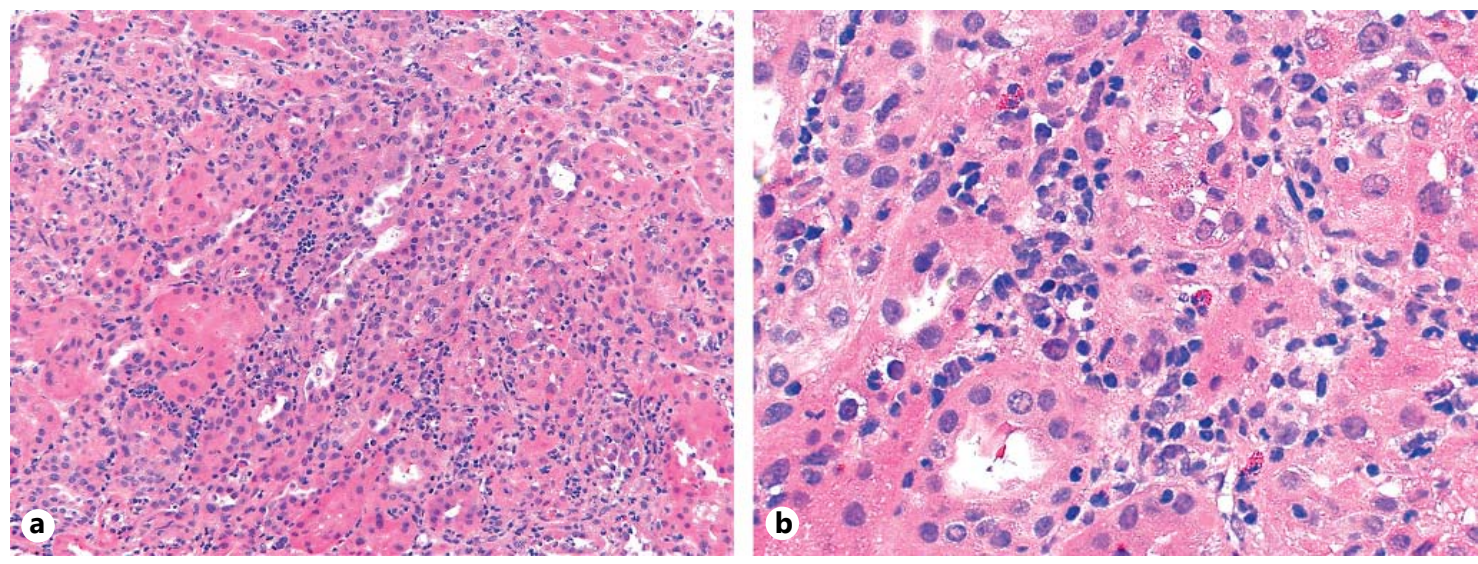

Fig. 3. a, b A 70-year-old Caucasian female who had completed chemotherapy 6 months ago for metastatic non-small cell lung cancer, 3 months ago was given a Pd1 inhibitor (Nivolumab), now presented with AKI where the baseline creatinine of 1.0 rose to $5.0 \mathrm{mg}$, urinalysis showed microhematuria and less than $1 \mathrm{~g}$ proteinuria with some white cell casts. There was no peripheral eo- sinophilia, rash or fever $\mathbf{a}, \mathbf{b} \mathrm{H} \& \mathrm{E}$ staining showing $\times 200$ and $\times 600$ magnification, respectively, illustrating renal cortical tissue showing diffuse, mild to moderate, active interstitial inflammation, mild edema, and frequent tubulitis and tubular epithelial injury, composed of activated lymphocytes, a few macrophages, and frequent eosinophils. py in patients in whom disease had progressed during or after one prior platinum-containing chemotherapy regimen. Among treatment-related select adverse events, $3 \%$ of the patients on nivolumab had an increase in creatinine as compared to $2 \%$ of the patients on docetaxel. Furthermore, one case of interstitial nephritis was also reported in the nivolumab group [9]. Regarding trials in patients with melanoma, both AKI and hyponatremia have been reported [37-43]. Online supplementary Table 3 summarizes the recent case reports of renal toxicities with this agent. Four cases were reported in 2 recent publications $[12,31]$. All 4 cases showed biopsy-proven AIN (most within 6-10 months following initiation of treatment). Figure 3 illustrates the pathology noted in a case of nivolumab-associated AIN. In summary, similar to pembrolizumab, AKI appears to develop late during the treatment phase, usually in the 6-12 months period. No gender preferences were noted and steroid treatment normalized renal function in $75 \%$ of the cases.

\section{Combined CTLA-4 and PD-1 Inhibitor Therapy}

Combination therapy with ipilimumab and nivolum$\mathrm{ab}$ appears to increase the incidence and severity of adverse events as compared to the use of nivolumab alone. In a randomized, double-blind phase 1 dose-escalation study, comparing nivolumab in combination with ipilimumab with standard-of-care ipilimumab monotherapy as a first-line treatment in patients with advanced melanoma, 4 adverse renal events among 94 patients that re- ceived the combination therapy were reported, including a grades 3-4 event. Three of them who were managed with immunomodulatory therapy achieved complete resolution. No renal events were reported in the ipilimumab monotherapy group [39]. In another phase 1 trial where these 2 drugs were combined in patients with advanced melanoma, and administered either concurrently or sequentially, a rise in creatinine was noted in 7 out of the 53 patients who received the concurrent therapy. Conversely, none of the patients who received the sequential therapy developed an adverse renal event [40]. In a randomized, double-blind, multicenter, phase 3 trial (CheckMate 067) that was conducted to evaluate the safety and efficacy of nivolumab alone or nivolumab combined with ipilimumab in comparison with ipilimumab alone in patients with previously untreated metastatic melanoma, 3 out of the $313(0.9 \%)$ patients treated with nivolumab alone developed a renal adverse event as compared to 17 out 313 (5\%) treated with ipilimumab and nivolumab and 8 out of 311 (2.5\%) treated with ipilimumab alone [41]. Cortazar et al. [12], Shirali et al. [31], and Murakami et al. [42] reported a total of 6 cases of ipilimumab and nivolumab combination therapy used in melanoma treatment, leading to biopsy-proven granulomatous or diffuse AIN. All were male, and 4 of the 6 patients had only partial recovery with steroids while 1 patient had complete recovery. The time frame ranged from 5 to 33 weeks. It appears that the injury might be more severe due to granuloma formation and perhaps 
Table 1. Common reported renal adverse events to the FDA adverse reporting database (FAERS) from 20113 rd quarter to 1 st quarter of 2015

\begin{tabular}{llllllrr}
\hline Drug name & $\begin{array}{l}\text { Renal } \\
\text { impairment* }\end{array}$ & Hypokalemia & Hyponatremia & Hypomagnesemia & Hyperkalemia & Hypophosphatemia & $\begin{array}{c}\text { Hypernatremia } \\
\text { Grand } \\
\text { total }\end{array}$ \\
\hline Ipilimumab & $\mathbf{2 2 0}$ & 64 & 159 & 8 & 19 & 13 & 4 \\
Nivolumab & $\mathbf{2 0}$ & 5 & 14 & 2 & 4 & 1 & 0 \\
Pembrolizumab & 16 & 3 & $\mathbf{1 9}$ & 0 & 1 & 0 & 0 \\
\hline
\end{tabular}

The numbers in the cell represent the total number of events of each category for the 3 immune check point inhibitors.

* Renal impairment comprises proteinuria, renal failure acute, acute kidney injury, elevated creatinine, hypercreatinemia, and nephritis.

** Bolded numbers represents most common reported reaction.

*** The search terms used for the FAERS database were "renal impairment, proteinuria, renal failure acute, acute kidney injury, elevated creatinine, hypercreatinemia nephritis, hyponatremia, hypokalemia, hypernatremia, hyperkalemia, hypophosphatemia, hypocalcemia, hypercalcemia, hypomagnesemia, and hypertension." There are important limitations with the FAERS database. The events are reported by providers and/or patients and there could be a reporting bias. In addition, not all demographic and comorbidity information is available to help identify if other nephrotoxic risk factors are present.

less responsive to steroids, and 1 patient required dialysis. Online supplementary Table 4 summarizes the renal effects of combined therapy.

\section{FDA Adverse Event Reporting System Database \\ Review of Renal Toxicities with ICI}

As part of this review, we evaluated all renal toxicities with the above-mentioned ICI reported to the FDA adverse event reporting system from the 3rd quarter of 2011 to the 2 nd quarter of 2015 . Table 1 summarizes our findings. Renal failure was the most commonly reported event followed by hyponatremia consistent with the reviewed literature findings. Renal failure and hyponatremia were also reported with PD-1 inhibitors. Ipilimumab has been in use since 2011 and PD-1 inhibitors since 2014 possibly confounding the increased rate of renal events seen with ipilimumab.

\section{Clinical Features and Mechanism of Injury}

While hematuria (16\%), eosinophilia (21\%), and worsening HTN (11\%) were noted in some patients, rising serum creatinine $(100 \%)$ and pyuria $(68 \%)[12,31]$ may be the only clinical clue in a large majority of the cases. As noted above, nephrotic syndrome is a rare finding in CTLA-4 antagonists.

CTLA-4 regulates peripheral tolerance by modulating the interaction between APC and T cells in secondary lymphoid organs. CTLA-4 deficiency was reported to trigger the early onset of severe lymphoproliferative autoimmune syndromes both in human and mouse via tissue self-antigen-specific $\mathrm{T}$-cell activation [1]. With CTLA-4 blockade, regulatory T cells (Tregs) lose their suppressive capacity and an uncontrolled activation of auto-reactive T cells occurs. Those cells then migrate and infiltrate the kidney. On the other hand, PD-1 contributes to tolerance primarily at the level of target organs. In the renal tissue, there is upregulation of PD-L1 by renal cells, which will bind and signal through PD-1 expressed by $\mathrm{T}$ cells, trying to prevent those cells from proliferating and damaging the tissue. However, when the self-reactive T cells have their PD-1 receptor blocked by the antibody, the PD-1/PD-L1 signaling will be interrupted and $\mathrm{T}$ cells will further proliferate and cause cytotoxic injury to the kidney [43]. In addition, PD-L1- and L2-deficient mice have also accelerated ischemic reperfusion renal injury [44]. PD-Ligands are essential in preventing inflammatory responses of the immune system in the target organs such as the kidney $[45,46]$. Also, PD1-knockout mice spontaneously developed glomerulonephritis $[47,48]$. The heterogeneity of the time course and delayed response are suggestive of a mechanism distinct from typical drug-induced AIN. It is possible that ICIs-induced AIN may be due to "reprogramming" of the immune system, leading to the loss of tolerance against endogenous kidney antigens, as opposed to a delayed type hypersensitivity reaction [12]. This might be why some of the cases had a long latency time period before AIN occurred. Thus, anti-PD-1 therapy may drive an autoimmune variant of interstitial nephritis, similar to the induction of autoimmune diabetes, possibly mediated by the loss of peripheral tolerance to self-reactive $\mathrm{T}$ cells [49]. The disruption of PD-1 signaling might also break tolerance to drug-specific effector $\mathrm{T}$ cells that are critical to the pathogenesis of AIN [50]. As a result, antiPD-1 therapy reactivates exhausted drug-specific T cells primed by the exposure to nephritogenic drugs, includ- 
Table 2. Renal effects of cytotoxic T-lymphocyte-associated protein 4 (CTLA-4) antagonists and the programmed death-1 (PD-1) inhibitors

\begin{tabular}{|c|c|c|}
\hline Agents & CTLA-4 antagonists (ipilimumab) & PD-1 inhibitors (nivolumab and pembrolizumab) \\
\hline $\begin{array}{l}\text { Mechanistic } \\
\text { differences }\end{array}$ & $\begin{array}{l}\text { 1. Limits T-cell response early in the immune response in } \\
\text { lymphoid tissues } \\
\text { 2. Expressed by T cells } \\
\text { 3. CTLA- } 4 \text { ligands expressed by antigen-presenting cells }\end{array}$ & $\begin{array}{l}\text { 1. Limits T-cell response later in the immune response, } \\
\text { primarily in peripheral tissues } \\
\text { 2. Expressed by T cells and other immune cells } \\
\text { 3. PD-1 ligands expressed by antigen-presenting cells and } \\
\text { other immune cells and can be inducibly expressed in } \\
\text { non-immune cells including tumor cells }\end{array}$ \\
\hline Onset of AIN & $\begin{array}{l}\text { AIN appears } 6-12 \text { weeks after initiation of therapy, with } \\
\text { longest duration being } 26 \text { weeks. Late onset associated with } \\
\text { more severe AKI requiring renal replacement therapy }\end{array}$ & AIN appears 3-12 months after initiation of therapy \\
\hline Gender & No gender preferences & No gender preferences \\
\hline $\begin{array}{l}\text { Electrolyte } \\
\text { disorders }\end{array}$ & $\begin{array}{l}\text { Hyponatremia cases related to hypophysitis (secondary } \\
\text { adrenal insufficiency) }\end{array}$ & Hyponatremia is rare \\
\hline Transplant & $\begin{array}{l}\text { In renal transplant patients, } 2 \text { cases reported no rejection } \\
\text { when given as a solo agent }\end{array}$ & $\begin{array}{l}\text { When given-patients had rejection especially following } \\
\text { use with CTLA- } 4 \text { inhibitors ( } 4 \text { cases reported), likely due } \\
\text { to loss of tolerance }\end{array}$ \\
\hline
\end{tabular}

AIN, acute interstitial nephritis.

** FDA approved, * in phase 2 or 3 clinical trials.

ing proton pump inhibitors and nonsteroidal anti-inflammatory drugs but subsequently inhibited by PD-1 signaling. Either scenario resulted in increased effector T-cell migration and function, leading to clinically significant renal injury. Shirali et al. [31] and Cortazar et al. [12] had the largest series of AIN reported with both the PD-1 inhibitors. In the Shirali et al. [31] study, all 6 patients ( 4 on nivolumab and 2 on pembrolizumab) were also on other drugs (proton pump inhibitors, nonsteroidal anti-inflammatory drugs) linked to AIN, but in most cases, the use of these drugs preceded anti-PD-1 antibody therapy. It is possible that PD-1 inhibitor therapy may release the suppression of T-cell immunity that normally permits renal tolerance to drugs known to be associated with AIN. Dual PD-1/CTLA-4 blockade synergistically might break the tolerance by unleashing the quiescent, tissue-specific, self-reactive T cells, which express high levels of PD-1. Table 2 summarizes the differences in the 2 classes of agents.

\section{ICIs in Kidney Transplantation}

Several cases of kidney injury have now been reported when the 3 agents discussed above were used in kidney transplant patients. Although Lipson et al. [51] had initially reported the successful administration of ipilimumab to 2 kidney transplantation patients with metastatic melanoma without any signs of rejection, they recently reported a case of tumor regression but allograft rejection after administration of pembrolizumab [52]. In addition, 3 cases of rejection were reported with the use of nivolum$\mathrm{ab}$ in kidney transplant patients with melanoma [53-55]. Online supplementary Table 5 summarizes all 6 cases. Based on the 6 cases, it appears that PD-1 inhibitors could be more prone to causing rejection in the transplanted kidney compared to CTLA-4 antagonists, especially when the patients have received anti CTLA-4 agents prior to PD-1 inhibitor treatment. This may be because PD-1-PD $\mathrm{L} 1$ interaction in the kidney tubular cells leads to the induction of FOXP3+ regulatory $\mathrm{T}$ cells, which play an es- 
sential role in maintaining graft tolerance and minimizing the chance of rejection [44]. In a recently published case [56], we presented a novel strategy to prevent rejection in the transplant patients receiving PD-1 inhibitors using preemptive steroids and sirolimus. The organ transplant community should be aware of the potential risk of rejection in kidney transplant recipients with the use of ICI.

\section{CKIN Recommendations}

In our opinion and based on available clinical trial data, AKI is a known complication of ICI. A renal consultation should be sought early and a kidney biopsy performed, if the risk associated with the procedure is low. All potential causes of AKI (pre renal, ATN, obstruction) should be evaluated. If AKI is confirmed on a kidney biopsy as AIN or a podocytopathy, we recommend the discontinuation of the checkpoint inhibitor and a course of corticosteroids. However, we cannot recommend a definitive dose and duration of steroid therapy. Based on cases reported, prednisone $1 \mathrm{mg} / \mathrm{kg}$ tapered over a period of 1-2 months may be sufficient. We additionally recommend close monitoring of the serum creatinine. Rechallenge with ICI therapy may be reasonable if other potentially offending agents (nonsteroidal anti-inflammatory drugs, proton pump inhibitors) are withdrawn and AIN has resolved. With this approach, serum creatinine should be monitored bi-weekly, with the reinstitution of steroids at the first sign of AKI from AIN without another cause. For patients on ipilimumab, renal function monitoring should be more frequent in the first 3 months, as the injury appears to happen earlier in the treatment course. For the PD-1 inhibitors, the injury usually happens later, so monitoring serum chemistries on a more frequent basis might be more prudent.

In kidney transplant recipients who develop malignancy post transplantation, these agents should be used with caution. It is important to consult with a transplant nephrologist before modifying immunosuppressive agents. The moderate reduction of immunosuppression may be warranted along with close monitoring of renal function. Conversion of tacrolimus to sirolimus and higher prednisone dose may be a reasonable treatment regimen to prevent rejection, and at the same time does not appear to minimize the efficacy of CTLA- 4 or PD-1 antibodies against malignancy. The authors reiterate that there are no substantial data available to support this preventive strategy and more evidence is needed. A close collaboration between oncologists, hematologists, and transplant nephrologists is strongly encouraged in this setting.

\section{Summary}

Checkpoint inhibitor-related renal toxicity is an immune-mediated process. While initial studies noted a small incidence of AKI (2-3\%), recent data suggest a higher incidence rate closer to $13-29 \%$ with ICI [12-14]. AIN is the most common biopsy finding reported. Ipilimumab has been associated with AIN and podocytopathies such as lupus like nephritis, minimal change disease, and TMA. Hyponatremia related to hypophysitis has been reported as well. The time of onset is 2-3 months in a majority of the cases. Most cases are responsive to steroids if identified early in the course of renal injury. Few patients may remain dialysis dependent. The renal injury related to anti PD-1 therapy is AIN. It usually appears later, 3-10 months into treatment. Steroids are also effective in the treatment of this immune-mediated adverse effect. When both CTLA-4 and PD-1 inhibitor drugs are combined, granulomatous or diffuse AIN can be found on kidney biopsy with partial response to steroids. While biopsy-proven interstitial nephritis related to these drugs is a complication associated with some degree of morbidity, one must keep in mind common causes of AKI in cancer patients such as volume depletion, dehydration, and sepsis. On the contrary, there is a potential for the under-recognition of nephritis due to the use of steroids for other non-renal complications such as dermatitis and colitis associated with these agents. Based on the 6 cases in the transplant literature, it appears that PD-1 inhibitors could be more prone to causing rejection in the transplanted kidney compared to CTLA-4 antagonists. This is especially true when the patients have received anti CTLA- 4 agents prior to PD-1 inhibitor treatment. These cases have been successfully managed with either discontinuation of the drug and simple observation and/or with use of systemic steroids. In organ transplant patients, a preventive strategy might be needed to minimize the chance of rejection when these agents are used for treatment of post-transplant cancers.

A close collaboration between oncologists, hematologists, and nephrologists is strongly encouraged. More investigation and database creation might be necessary to better understand the mechanism behind the renal disease associated with ICIs.

\section{Disclosure Statement}

The results presented in this paper have not been published previously in whole or part, except in the abstract format.

This work was part of a C-KIN working group on ICIs-related renal toxicities. V.L.-V. and G.D. have received unrestricted edu- 
cational grants and honoraria from Roche for last 3 years. V.L.-V. received unrestricted educational grants from Amgen, Leo Pharma, Merck, Pierre Fabre Oncologie, Takeda, Teva for the last 3 years. K.D.J. serves on the American Society of Nephrology Onconephrology Forum. V.L.-V. is the current President of C-KIN. G.D. and K.D.J. serve on the Governing Body of C-KIN and R.W. is an expert member of C-KIN.

\section{Acknowledgment}

We thank Dr. Matthew A. Sparks, Duke University for his critical review of the manuscript. We thank Dr. Surya V. Seshan, Weill Cornell Medical Center for providing the pathology figures found in this manuscript.

\section{References}

1 Iannello A, Thompson TW, Ardolino M, Marcus A, Raulet DH: Immunosurveillance and immunotherapy of tumors by innate immune cells. Curr Opin Immunol 2016;38:52-58.

2 Smyth MJ, Dunn GP, Schreiber RD: Cancer immunosurveillance and immunoediting: the roles of immunity in suppressing tumor development and shaping tumor immunogenicity. Adv Immunol 2006;90:1-50.

-3 Zitvogel L, Tesniere A, Kroemer G: Cancer despite immunosurveillance: immunoselection and immunosubversion. Nat Rev Immunol 2006;6:715-727.

-4 Barbee MS, Ogunniyi A, Horvat TZ, Dang TO: Current status and future directions of the immune checkpoint inhibitors ipilimumab, pembrolizumab, and nivolumab in oncology. Ann Pharmacother 2015;49:907-937.

5 Luke JJ, Ott PA: PD-1 pathway inhibitors: the next generation of immunotherapy for advanced melanoma. Oncotarget 2015;6:34793492.

6 Terme M, Ullrich E, Aymeric L, Meinhardt K, Desbois M, Delahaye N, et al: IL-18 induces PD-1-dependent immunosuppression in cancer. Cancer Res 2011;71:5393-5399.

-7 Tang PA, Heng DY: Programmed death 1 pathway inhibition in metastatic renal cell cancer and prostate cancer. Curr Oncol Rep 2013;15:98-104.

8 Borghaei H, Paz-Ares L, Horn L, Spigel DR, Steins M, Ready NE, et al: Nivolumab versus docetaxel in advanced nonsquamous nonsmall-cell lung cancer. N Engl J Med 2015; 373:1627-1639.

-9 Brahmer J, Reckamp KL, Baas P, Crinò L, Eberhardt WE, Poddubskaya E, et al: Nivolumab versus docetaxel in advanced squamous-cell non-small-cell lung cancer. N Engl J Med 2015;373:123-135.

10 Garon EB, Rizvi NA, Hui R, Leighl N, Balmanoukian AS, Eder JP, et al: Pembrolizumab for the treatment of non-small-cell lung cancer. N Engl J Med 2015;372:2018-2028.

11 FDA Adverse Event Reporting System (FAERS). http://www.fda.gov/Drugs/Guidan ceComplianceRegulatoryInformation/Surveillance/AdverseDrugEffects/default.htm (accessed March 18, 2016).

12 Cortazar FB, Marrone KA, Troxell ML, Ralto KM, Hoenig MP, Brahmer JR, et al: Clinicopathological features of acute kidney injury associated with immune checkpoint inhibitors. Kidney Int 2016;90:638-647.
13 Mason NT, Khushalani NI, Weber JS, Antonia SJ, McLeod HL: Modeling the cost of immune checkpoint inhibitor-related toxicities. J Clin Oncol 2016;34(suppl):abstr 6627.

14 Hirsch J, Wanchoo R, Devoe C, Jhaveri KD: Incidence of AKI in immune checkpoint inhibitors, single center study. J Am Soc Nephrol 2016;27:763.

15 Voskens CJ, Goldinger SM, Loquai C, Robert C, Kaehler KC, Berking C, et al: The price of tumor control: an analysis of rare side effects of anti-CTLA-4 therapy in metastatic melanoma from the ipilimumab network. PLoS One 2013;8:e53745.

16 Thajudeen B, Madhrira M, Bracamonte E, Cranmer LD: Ipilimumab granulomatous interstitial nephritis. Am J Ther 2015;22:e84-e87.

17 O’Day SJ, Maio M, Chiarion-Sileni V, Gajewski TF, Pehamberger $\mathrm{H}$, Bondarenko IN, et al: Efficacy and safety of ipilimumab monotherapy in patients with pretreated advanced melanoma: a multicenter single-arm phase II study. Ann Oncol 2010;21:1712-1717.

18 Forde PM, Rock K, Wilson G, O’Byrne KJ: Ipilimumab-induced immune-related renal failure - a case report. Anticancer Res 2012; 32:4607-4608.

19 Fadel F, El Karoui K, Knebelmann B: AntiCTLA4 antibody-induced lupus nephritis. N Engl J Med 2009;361:211-212.

20 Voskens C, Cavallaro A, Erdmann M, Dippel O, Kaempgen E, Schuler G, et al: Anti-cytotoxic T-cell lymphocyte antigen-4-induced regression of spinal cord metastases in association with renal failure, atypical pneumonia, vision loss, and hearing loss. J Clin Oncol 2012;30:e356-e357.

21 Izzedine H, Gueutin V, Gharbi C, Mateus C, Robert C, Routier E, et al: Kidney injuries related to ipilimumab. Invest New Drugs 2014; 32:769-773.

22 Kidd JM, Gizaw AB: Ipilimumab-associated minimal-change disease. Kidney Int 2016;89: 720.

23 Barnard ZR, Walcott BP, Kahle KT, Nahed BV, Coumans JV: Hyponatremia associated with Ipilimumab-induced hypophysitis. Med Oncol 2012;29:374-377.

24 Chodakiewitz Y, Brown S, Boxerman JL, Brody JM, Rogg JM: Ipilimumab treatment associated pituitary hypophysitis: clinical presentation and imaging diagnosis. Clin Neurol Neurosurg 2014;125:125130.
25 Pembrolizumab package insert. http://www. accessdata.fda.gov/drugsatfda_docs/ label/2014/125514lbl.pdf(lastaccessed January 1,2016).

26 Patnaik A, Kang SP, Rasco D, Papadopoulos KP, Elassaiss-Schaap J, Beeram M, et al: Phase I study of pembrolizumab (MK-3475; AntiPD-1 monoclonal antibody) in patients with advanced solid tumors. Clin Cancer Res 2015; 21:4286-4293.

27 Robert C, Ribas A, Wolchok JD, Hodi FS, Hamid O, Kefford R, et al: Anti-programmeddeath-receptor-1 treatment with pembrolizumab in ipilimumab-refractory advanced melanoma: a randomised dose-comparison cohort of a phase 1 trial. Lancet 2014;384: 1109-1117.

28 Ribas A, Puzanov I, Dummer R, Schadendorf D, Hamid O, Robert C, et al: Pembrolizumab versus investigator-choice chemotherapy for ipilimumab-refractory melanoma (KEYNOTE-002): a randomised, controlled, phase 2 trial. Lancet Oncol 2015; 16:908-918.

29 Herbst RS, Baas P, Kim DW, Felip E, PérezGracia JL, Han JY, et al: Pembrolizumab versus docetaxel for previously treated, PD-L1positive, advanced non-small-cell lung cancer (KEYNOTE-010): a randomised controlled trial. Lancet 2016;387:1540-1550.

30 Min L, Hodi FS: Anti-PD1 following ipilimumab for mucosal melanoma: durable tumor response associated with severe hypothyroidism and rhabdomyolysis. Cancer Immunol Res 2014;2:15-18.

31 Shirali AC, Perazella MA, Gettinger S: Association of acute interstitial nephritis with programmed cell death 1 inhibitor therapy in lung cancer patients. Am J Kidney Dis 2016; 68:287-291.

32 Nivolumab Package Insert. http://www.access data.fda.gov/drugsatfda_docs/label/2014/ 125554lbl.pdf (last accessed January 1, 2016).

-33 Topalian SL, Sznol M, McDermott DF, Kluger HM, Carvajal RD, Sharfman WH, et al: Survival, durable tumor remission, and longterm safety in patients with advanced melanoma receiving nivolumab. J Clin Oncol 2014;32:1020-1030.

34 Eigentler TK, Hassel JC, Berking C, Aberle J, Bachmann O, Grünwald V, et al: Diagnosis, monitoring and management of immune-related adverse drug reactions of anti-PD- 1 antibody therapy. Cancer Treat Rev 2016;45:7-18. 
35 Gettinger SN, Horn L, Gandhi L, Spigel DR, Antonia SJ, Rizvi NA, et al: Overall survival and long-term safety of nivolumab (anti-programmed death 1 antibody, BMS-936558, ONO-4538) in patients with previously treated advanced non-small-cell lung cancer. J Clin Oncol 2015;33:2004-2012.

36 Rizvi NA, Mazières J, Planchard D, Stinchcombe TE, Dy GK, Antonia SJ, Horn L, et al: Activity and safety of nivolumab, an antiPD-1 immune checkpoint inhibitor, for patients with advanced, refractory squamous non-small-cell lung cancer (CheckMate 063): a phase 2, single-arm trial. Lancet Oncol 2015; 16:257-265.

-37 Topalian SL, Sznol M, McDermott DF, Kluger $\mathrm{HM}$, Carvajal RD, Sharfman WH, et al: Survival, durable tumor remission, and longterm safety in patients with advanced melanoma receiving nivolumab. J Clin Oncol 2014;32:1020-1030.

- 38 Weber JS, D'Angelo SP, Minor D, et al: Nivolumab versus chemotherapy in patients with advanced melanoma who progressed after anti-CTLA-4 treatment (CheckMate 037): a randomised, controlled, open-label, phase 3 trial. Lancet Oncol 2015;16:375-384

-39 Postow MA, Chesney J, Pavlick AC, Robert C, Grossmann K, McDermott D, et al: Nivolum$\mathrm{ab}$ and ipilimumab versus ipilimumab in untreated melanoma. N Engl J Med 2015;372: 2006-2017.

40 Wolchok JD, Kluger H, Callahan MK, Postow MA, Rizvi NA, Lesokhin AM, et al: Nivolumab plus ipilimumab in advanced melanoma. $\mathrm{N}$ Engl J Med 2013;369:122-133.
41 Larkin J, Hodi FS, Wolchok JD: Combined nivolumab and ipilimumab or monotherapy in untreated melanoma. N Engl J Med 2015; 373:23-34.

42 Murakami N, Borges T, Yamashita M, Riella L: Severe acute interstitial nephritis after combination immune-checkpoint inhibitor therapy for metastatic melanoma. Clin Kidney J 2016;9:411-417.

43 Vandiver JW, Singer Z, Harshberger C: Severe hyponatremia and immune nephritis following an initial infusion of nivolumab. Target Oncol 2016;11:553-556.

44 Riella LV, Paterson AM, Sharpe AH, Chandraker A: Role of the PD-1 pathway in the immune response. Am J Transplant 2012;12: 2575-2587.

45 Jaworska K, Ratajczak J, Huang L, Whalen K, et al: Both PD-1 ligands protect the kidney from ischemia reperfusion injury. J Immunol 2015; 194:325-333.

46 Menke J, Lucas JA, Zeller GC, et al: Programmed death 1 ligand (PD-L) 1 and PD-L2 limit autoimmune kidney disease: distinct roles. J Immunol 2007;179:7466-7477.

47 Waeckerle-Men Y, Starke A, Wuthrich RP: PD-L1 partially protects renal tubular epithelial cells from the attack of CD8+ cytotoxic T cells. Nephrol Dial Transplant 2007;22:15271536.

48 Nishimura H, Nose M, Hiai H, Minato N, Honjo T: Development of lupus-like autoimmune diseases by disruption of the PD-1 gene encoding an ITIM motif-carrying immunoreceptor. Immunity 1999;11:141-151.
49 Hughes J, Vudattu N, Sznol M, et al: Precipitation of autoimmune diabetes with antiPD-1 immunotherapy. Diabetes Care 2015; 38:e55-e57.

50 Spanou Z, Keller M, Britschgi M, et al: Involvement of drug-specific $T$ cells in acute drug-induced interstitial nephritis. J Am Soc Nephrol 2006;17:2919-2927.

51 Lipson EJ, Bodell MA, Kraus ES, Sharfman WH: Successful administration of ipilimumab to two kidney transplantation patients with metastatic melanoma. J Clin Oncol 2014; 32:e69-e71.

52 Lipson EJ, Bagnasco SM, Moore J Jr, Jang S, Patel MJ, Zachary AA, et al: Tumor regression and allograft rejection after administration of anti-PD-1. N Engl J Med 2016;374: 896-898.

53 Alhamad T, Venkatachalam K, Linette GP, Brennan DC: Checkpoint inhibitors in kidney transplant recipients and the potential risk of rejection. Am J Transplant 2016;16:13321333.

54 Spain L, Higgins R, Gopalakrishnan K, Turajlic S, Gore M, Larkin J: Acute renal allograft rejection after immune checkpoint inhibitor therapy for metastatic melanoma. Ann Oncol 2016;27:1135-1137.

55 Boils CL, Aljadir DN, Cantafio AW: Use of the PD-1 pathway inhibitor nivolumab in a renal transplant patient with malignancy. Am J Transplant 2016;16:2496-2497.

56 Barnett R, Barta VS, Jhaveri KD: Preserved renal allograft function while using the PD-1 pathway inhibitor nivolumab. N Engl J Med 2017;376:191-192. 